Article

\title{
Improvement of the Sphericity and the Thickness Uniformity of the Polystyrene (PS) Shell Microsphere during Curing Process
}

\author{
Xiaotian Han ${ }^{1}$, Hua Zhou ${ }^{2}$, Yifei Zhu ${ }^{3}$, Liangyu Wu ${ }^{1}$, Feng Yao ${ }^{2, *}$ (i) and Cheng $\mathrm{Yu}^{1,3, *}$ \\ 1 School of Hydraulic, Energy and Power Engineering, Yangzhou University, Yangzhou 225127, China; \\ xthan@microflows.net (X.H.); lywu@yzu.edu.cn (L.W.) \\ 2 Jiangsu Key Laboratory of Micro and Nano Heat Fluid Flow Technology and Energy Application, School of \\ Environmental Science and Engineering, Suzhou University of Science and Technology, Suzhou 215009, \\ China; hzhou@microflows.net \\ 3 School of Energy and Environment, Southeast University, Nanjing, Jiangsu 210096, China; \\ yfzhu@microflows.net \\ * Correspondence: yaofeng@usts.edu.cn (F.Y.); iamyucheng@seu.edu.cn (C.Y.); Tel.: +86-514-8797-1315 (C.Y.)
}

Received: 14 April 2019; Accepted: 13 June 2019; Published: 14 June 2019

\begin{abstract}
To improve the quality of dispersed polystyrene (PS) compound droplets, a new random rotating curing system is designed. In addition, the qualities of the curing products of the PS compound droplets of this new system are compared with those of the traditional curing system with a constant rotating speed, so as to verify the effectiveness of the new system on the quality improvement of the PS compound droplets. The effect of the liquid level, rotation rate and the density difference on the curing process is also analyzed to reveal the mechanism of the curing process in a rotating flow field. The results indicate that, in the new rotating curing system, the disturbance of the fluid increases the deformation recovery ability of the compound droplets. Furthermore, the vortex with different directions in the external flow fields, make the compound droplets spin in many directions, which improves the spheroidization and concentricity of the compound droplets. Compared with using the traditional rotating curing system, when utilizing the random rotating curing system, the sensitivity of the microspheres' quality to the density mismatch between the phases is smaller, and the sphericity and the thickness uniformity of the polystyrene (PS) microsphere increase by $10.2 \%$ and $4.5 \%$, respectively. In addition, there is an optimal rotation rate for the random rotating curing device, which can optimize the survival rate and quality of the hollow microspheres.
\end{abstract}

Keywords: microencapulation; polystyrene microsphere; compound droplets; external flow fields; curing process

\section{Introduction}

Microencapsulation with polymer materials is an effective way to protect the encapsulated material and control its release to the external environment, and it has been widely used for micro reactors $[1,2]$, bioassays [3], controlled releases [4,5], and inertial confinement fusion (ICF) [6,7]. In indirect-driven ICF experiments, a microsphere is filled with deuterium (DD) or deuterium-tritium (DT) fuel, and then placed in a metal cylindrical hohlraum. The laser deposits its power inside the hohlraum, and the laser power is converted to X-ray. Then, the fuel gas is compressively heated by the X-ray to drive the implosion, which initiates the fusion reaction [8-10]. It has been reported that the sphericity and uniformity of the wall thickness of the microsphere are important to the symmetry and hydrodynamic stability in the implosion, as well as to the success of the ICF [11-13]. Therefore, it is crucial to fabricate the microsphere for ICF with a high-quality precision. 
Recently, in indirect-driven ICF, hollow microspheres with a polystyrene (PS) shell are widely used, fabricated from the compound droplets through a curing process [14,15]. For the curing process of compound droplets by emulsion microencapsulation, a substantial amount of research has been conducted, mainly focused on the theoretical and experimental study of the effects of interfacial tension, density matching and the diffusion rate on the sphericity and thickness uniformity of compound droplets [16,17]. To improve the quality of the target microspheres, Takagi et al. [18] have successfully prepared different sizes of polystyrene hollow microspheres by reducing the density difference between the milk particles and the external water phase. The influence of the density matching, stirring rate and concentration of the organic solvent in the external aqueous solution on the spherical deviation of $1 \mathrm{~mm}$ poly-alpha methylstyrene (PAMS) microspheres was described in detail by McQuillan $[19,20]$. The results show that, with the increase of the microsphere size, the density matching between the internal water phase, the oil phase and the external water phase must be improved. Liu et al. [21] studied the effect of the interfacial tension on the quality of PS microspheres. By using polyacrylic acid (PAA) instead of polyvinyl alcohol (PVA), the interfacial tension between the milk particles and the external water phase was increased, and the spheroidization of the compound droplets was strengthened to improve the quality of the solidified microspheres. The rotating curing system is mostly applied to the curing process of the compound droplets [22,23]. However, the detailed flow field inside the rotating container, as well as the effects of the rotation rate and the liquid level on the quality of the microspheres, are less understood.

Moreover, the traditional rotating curing system only rotates around the axis in the center, which is sensitive to the density mismatch and may reduce the quality of the microspheres [24,25]. However, there are stringent specifications on the sphericity and wall thickness uniformity of microspheres for ICF, since asymmetry and hydrodynamic instability would be exponentially amplified by any deviations from a perfect sphericity, disrupting the ablation-driven implosion. The qualities of the microspheres fabricated from the traditional rotating curing system are not good enough for the high standard of the target for the ICF. Therefore, it is urgent to design a new rotating curing system with various rotating directions to improve the qualities of the curing products of the PS compound droplets. In this paper, a new random rotating curing system is designed, and the curing process is experimentally studied and compared with the traditional curing system. The effect of the liquid level, rotation rate and the density difference on the curing process is also analyzed to reveal the mechanism of the curing process in a rotating flow field. In addition, the optimization of the rotation rate for the random rotating curing system is carried out to improve the sphericity and the thickness uniformity of the microsphere.

\section{Experimental Section}

\subsection{Preparation System}

As shown in Figure 1, a coaxial microfluidics device is used in this experiment to produce polystyrene-in-oil compound droplets. Each phase is driven by an injection pump, and the preparation of different sizes of compound droplets can be achieved by changing the flow rate of each phase. For the formation of the compound droplets, the deionized water is used as the inner phase fluid, the fluorobenzene $(10 \mathrm{wt} \%)$ solution is used as the intermediate phase fluid, and the PVA ( $2 \mathrm{wt} \%)$ solution is used as the outer phase fluid.

Figure 2 shows the schematic diagram of two curing devices used in this experiment. As shown in Figure $2 \mathrm{a}$, the traditional curing system rotates around the center shaft in one direction with a constant speed, where the diameter and length of the container are $D$ and $L$, respectively, and where the liquid level is $H$. As shown in Figure $2 b$, the random rotating curing system drives the container to rotate by means of the four axis ball grinding apparatus. The four axes are symmetrically distributed in space and intersect at the center of the spherical vessel. The included angle between any two axes is $109.47^{\circ}$, and the angular velocity of each axis is equal, but the direction is switched 6 times in one 
cycle, as shown in Figure 3. At any time, there are only two revolving forward rotations, and the two rotating axes rotate backward.

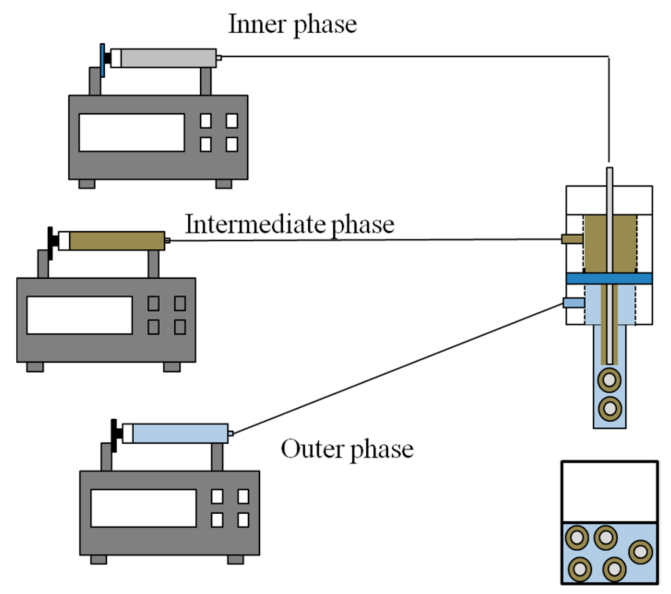

Figure 1. Schematic diagram of the preparation system.

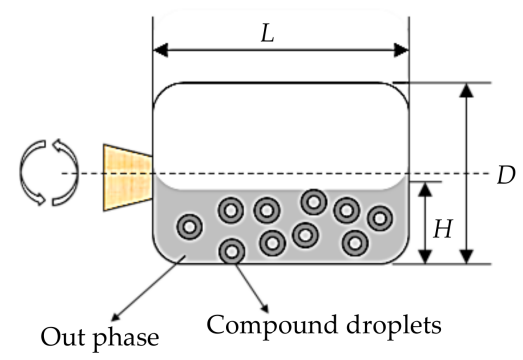

(a) Traditional curing system.

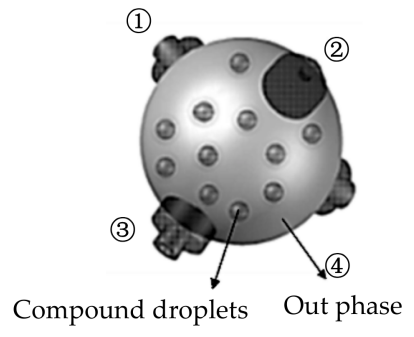

(b) Random rotating curing system.

Figure 2. Schematic diagram of the curing system. (a) In the traditional curing system, the container rotates in one direction around the center shaft. The lengths of the container are $D$ and $L$, respectively, and the liquid level is $H$; (b) In the random rotating curing system, the rotation of the container is controlled by the four axis ball grinding apparatus (1), (2), (3) and (4).

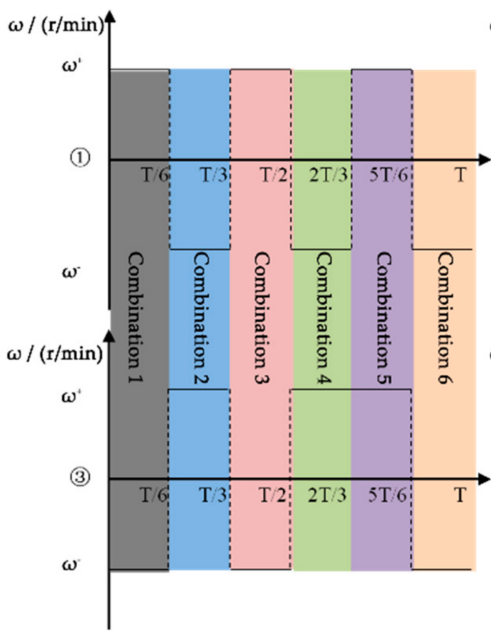

(a)

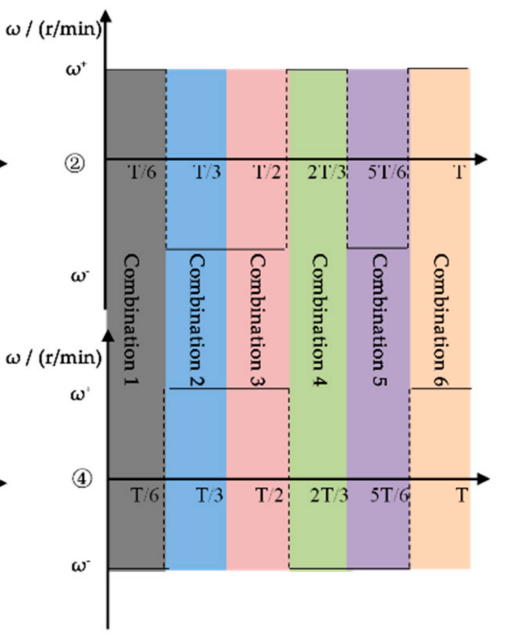

(b)

Figure 3. Diagrammatic sketch of the switch combination of the rotating shaft: $\omega+$ and $\omega$ - mean the forward rotation and backward rotation. (a) theoretical random rotation; (b) practical random rotation.

The motion trajectories of 6 combinations of spherical curing vessels were simulated by means of the MATLAB software (R2015a, MathWorks, Natick, MA, USA), and the results are shown in Figure 4. 
The rotation trajectory covers some part of the spherical surface under each combination, and the spherical curing vessel moves almost evenly across the surface of the sphere under the six combinations, which guarantees the symmetry of the rotation trajectory and the relative uniformity of the time.

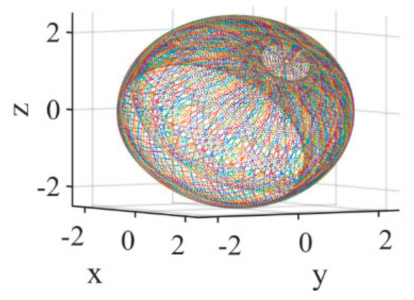

(a)

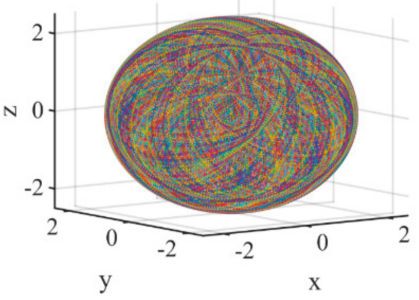

(b)

Figure 4. Simulation diagram of the motion trajectories. (a) Combination 1 ; (b) Total combination.

\subsection{Measurement System}

The internal diameter $D$ and wall thickness $t$ of the PS microspheres were measured from six equal points of the microspheres, at 0, 60, 120, 180, 240 and 300 degrees, by the coordinate measuring machine (VMR 3020, Nikon, Tokyo, Japan). Nikon VMR 3020 is used to determine the outer contour of the PS particle, including the size and sphericity. The wall thickness of the PS particle and its uniformity are measured based on the high-resolution X-ray radiography via an image processing program, as shown in Figure 5. We first obtained the original X-ray image of the simple PS microsphere (see Figure 5a) and converted it into a binary image that only contains two types of pixels, 0 and 1 ( 0 is the inner and outer area of the PS microsphere, and 1 is the coverage area of the microsphere shell) (see Figure $5 b$ ). Then, we programmatically obtain two best fitted circles to characterize the inner and outer profiles of the binary image, when the minimum sum of the squared errors occurs between the pixel data of the fitting circles and the shell profiles of the binary image. In our study, different X-ray images will be taken at six random discrete positions for one PS microsphere, and the above image analysis procedure is repeated for every $\mathrm{X}$-ray image. Finally, the average relative deviation $\Delta$ between the best fitted outer circle and the pixel data is obtained from six X-ray images of a PS microsphere, and the wall thickness of the PS microsphere, $t$, can also be gained as the average difference between the fitted outer and inner circles. The definitions of sphericity and uniformity are as follows:

$$
\begin{gathered}
\text { Sphericity }=1-\left(D_{\max }-D_{\min }\right) / D \\
\Delta t=t_{\max }-t_{\min }
\end{gathered}
$$

$D_{\max }, D_{\min }$ and $\bar{D}$ are the maximum diameter, minimum diameter and average diameter, respectively, of the six equal points of the microspheres, and $t_{\max }$ and $t_{\min }$ are the maximum and minimum wall thicknesses, respectively, at the six equal points of the microspheres. The densities of the water and the microsphere are measured by the digital densitometer (DMA ${ }^{\text {TM }} 5000$ M, Anton Paar, Graz, Austria), which has a measurement accuracy of $\pm 7 \times 10^{-5} \mathrm{~g} / \mathrm{cm}^{3}$.
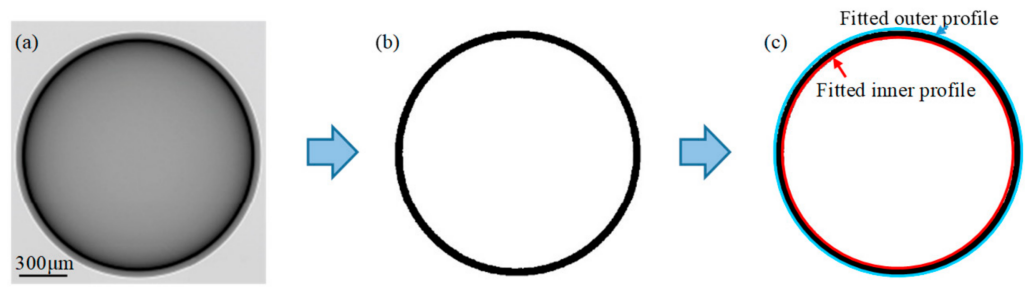

Figure 5. Schematic of the measurement of the wall thickness and the sphericity of the PS microsphere by X-ray in one direction: (a) original X-ray image, (b) binary image, and (c) fitted inner and outer profiles of (b). 


\section{Results and Discussion}

\subsection{Experimental Parameters of the Traditional Curing System}

For the curing container used in the traditional rotary curing device, the external flow field is determined by the rotation rates and liquid levels, and it strongly affects the quality of the compound droplets. The experiments in this paper are designed for different liquid levels $(H=D / 5, H=D / 3$, $H=D / 2$, and $H=3 D / 4$ ). At the same level, the rotation rates are 20,30, 40, 50 and $60 \mathrm{r} / \mathrm{min}$, respectively. In this section, the PVA ( $2 \mathrm{wt} \%$ ) solution is used as the outer phase fluid. The experimental hollow microsphere samples were measured by an X-ray machine, and the results are shown in Figure 6.

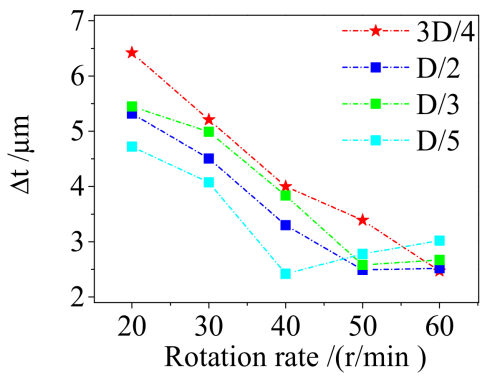

(a)

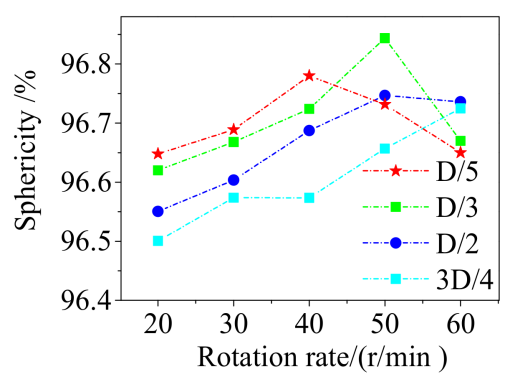

(b)

Figure 6. The effects of the rotation rate and liquid level on the quality of the hollow microspheres. (a) Uniformity of wall thickness; (b) Sphericity.

The results show that with the increase in the rotation rate, the sphericity of the hollow microspheres and the uniformity of the wall thickness first increase and then decrease with the increase in the rotation rate. This can be explained by the fact that the shear force produced by the external flow field is smaller at the lower rotation rate, and that the deformation of the compound droplets is more sensitive to the shear force. A higher rotation rate can improve the quality of the hollow microspheres. When the rotation rate increases again, the shear force is so large that the deformation of the compound droplets cannot be recovered in a short time, which results in the decrease of the sphericity and the uniformity of the thickness of the hollow microspheres. When the liquid level exceeds a certain value, the sphericity and wall thickness uniformity increase with the increase in the rotation rate. Due to the increase in the liquid level, the vortex generated in the container moves to the center, and the smaller shear effect of the central area provides a more suitable condition for improving the quality of the hollow microspheres.

It can be seen from the experimental results that there is an optimum rotation rate for different liquid levels, and that the optimum rotation rate is increased with the increase in the liquid level. When the level is $D / 5$, the optimum rotation rate is $40 \mathrm{r} / \mathrm{min}$, and the optimum rotation rate is $50 \mathrm{r} / \mathrm{min}$ when the level is increased to $D / 3$ and $D / 2$. However, when the liquid level increases to $3 D / 4$, there the optimum rotation rate does not exist, because the high liquid level will move the vortex to the center and a higher rotation rate is needed to achieve the best shear strength.

\subsection{Traditional Rotating Curing Device and Random Rotating Curing Device}

To study the influence of the external flow fields on the quality of the hollow microspheres, three experiments with various density differences are designed by changing the components of the external water phase. The density differences between the external water phase and the compound droplets are $\Delta \rho_{1}=0.0004 \mathrm{~g} / \mathrm{cm}^{3}, \Delta \rho_{2}=0.0044 \mathrm{~g} / \mathrm{cm}^{3}$ and $\Delta \rho_{3}=0.0068 \mathrm{~g} / \mathrm{cm}^{3}$, respectively. To achieve various density differences, PVA (2 wt \%) + calcium chloride (0.5 wt \%) solution, PVA (2 wt \%) solution, and PVA $(0.5 \mathrm{wt} \%)$ solution are used as the outer phase fluid. In addition, the three curing modes used in the experiment are shown in Figure 7. Among these modes, Mode 1 and Mode 2 use the traditional rotary curing device; the external water phases of the containers in Mode 1 and Mode 2 are fully filled 
and partially filled, respectively. Mode 3 uses the random rotating device, and the water phase in the container is fully filled.

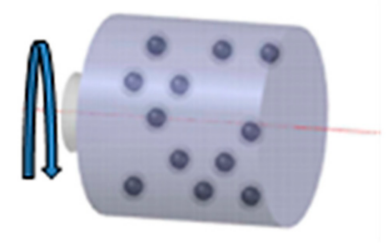

(a)

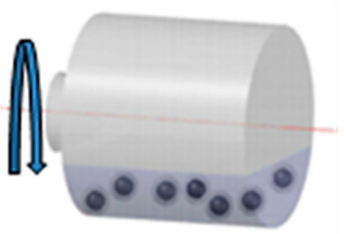

(b)

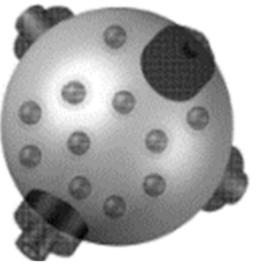

(c)

Figure 7. Schematic diagram of the three curing modes. (a) Mode 1; (b) Mode 2; (c) Mode 3.

Figures 8 and 9 show the effects of curing modes on the sphericity and wall thickness uniformity of the hollow microspheres under different density difference conditions. The experimental results in Figure 8 show that the sphericity of the hollow microspheres increases with the decrease in the density difference under the same curing mode. Due to the decrease in the density difference, the movement of the compound droplets easily follows the movement of the external water phase, which helps the compound droplets to spin in the external flow field and enhances the self-deformation of the compound droplets.

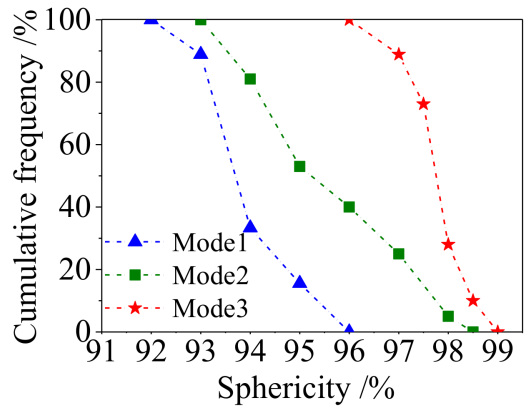

(a)

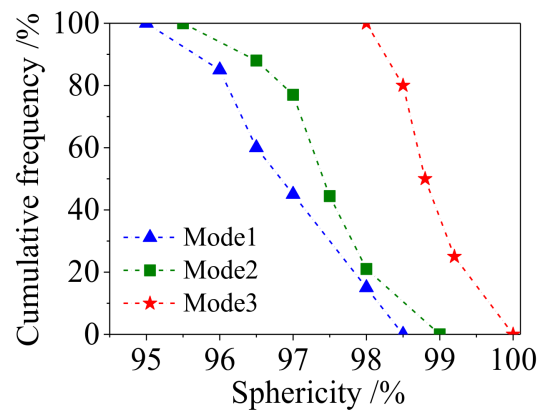

(b)

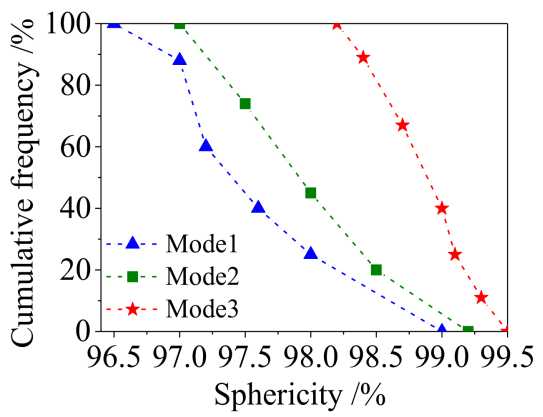

(c)

Figure 8. Effect of the curing modes on the sphericity. (a) $\Delta \rho=0.0068 \mathrm{~g} / \mathrm{cm}^{3} ;$ (b) $\Delta \rho=0.0044 \mathrm{~g} / \mathrm{cm}^{3}$;

(c) $\Delta \rho=0.0004 \mathrm{~g} / \mathrm{cm}^{3}$.

In addition, the sensitivity of the curing modes to the density difference is different. Mode 1 is the most sensitive to the density difference. When the density difference decreases from $\Delta \rho_{3}=0.0068 \mathrm{~g} / \mathrm{cm}^{3}$ to $\Delta \rho_{1}=0.0004 \mathrm{~g} / \mathrm{cm}^{3}$, the sphericity of the microspheres increases from $93 \%$ to $97 \%$, while the sensitivity of Mode 3 to the density difference is the weakest. The sphericity of the microspheres only increases from $96.5 \%$ to $98 \%$. The sensitivity of Mode 2 is between the two curing modes. Similarly, Figure 9 shows that the effects of the external flow fields on the thickness uniformity and sphericity of the hollow microspheres are the same. Generally, under the density mismatch condition, the thickness 
uniformity and sphericity of the hollow microspheres utilizing the random rotating curing system are $10.2 \%$ and $3.5 \%$ higher, respectively, than those utilizing the traditional random rotating curing system.

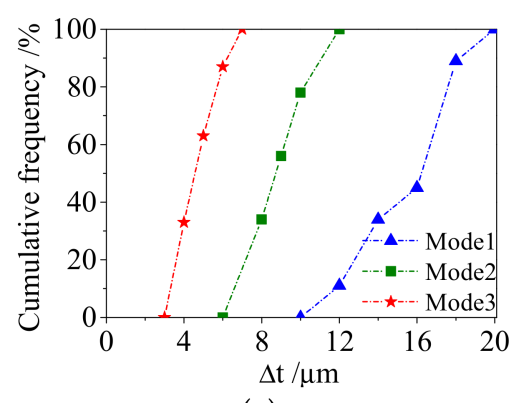

(a)

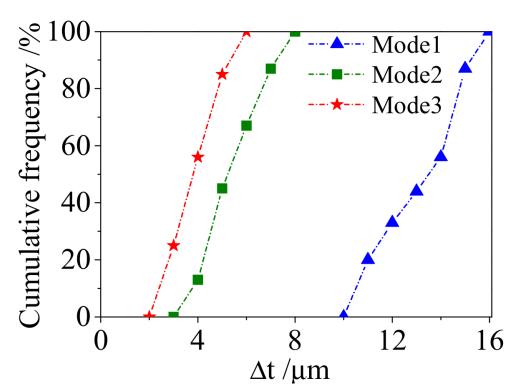

(b)

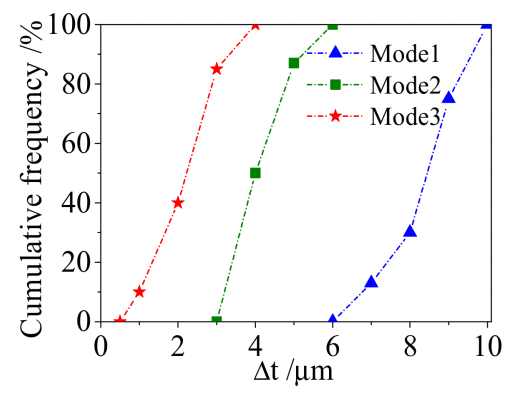

(c)

Figure 9. Effect of the curing modes on the thickness uniformity. (a) $\Delta \rho=0.0068 \mathrm{~g} / \mathrm{cm}^{3} ;$ (b) $\Delta \rho=0.0044 \mathrm{~g} / \mathrm{cm}^{3}$; (c) $\Delta \rho=0.0004 \mathrm{~g} / \mathrm{cm}^{3}$.

It is known from the experimental results that the Mode 3 random rotating device is more conducive to the curing of compound droplets, which is due to the curing flow field created by the random rotary device achieving a three-dimensional irregular circumferential shear effect of the external flow field on the compound droplets, overcoming the problem of emulsion eccentricity and demulsification caused by the difference of the floating lift caused by the density mismatch.

In the actual curing process of the compound droplets, the movement of the compound droplets in the Mode 1 flow field is approximated to be a uniform circumference, which makes the compound droplets and external flow fields remain relatively stationary. The centripetal-force-driven deformation of the compound droplets cannot self-recover through its own motion. Therefore, in Mode 1, the sphericity and thickness uniformity of the hollow microspheres are difficult to improve. In Mode 2, the shear force produced by the external flow field drives the compound droplets to move with the external flow field, which is beneficial for the restoration of the deformation. In addition, the existence of a vortex is beneficial to the spin of the compound droplets. The sphericity and concentricity of the curing hollow microspheres are improved. For Mode 3, the disturbance of the fluid increases the deformation recovery ability of the compound droplets. Furthermore, the vortex with different directions in the external flow field makes the compound droplets spin in many directions, which improves the spheroidization and concentricity of the compound droplets. The hollow microspheres cured by different curing modes are shown in Figure 10. To better compare the traditional rotating curing device and the random rotating curing device, the merit factor of the microsphere is defined as:

$$
M=\text { Sphericity } \times(1-\Delta t / \bar{D})
$$

Figure 11 shows the merit factor of the microsphere for different modes. It is evident that Mode 3 is the best mode for the curing process. The merit factor in Mode 3 is the highest whatever the density difference is and is less sensitive to the density difference. 


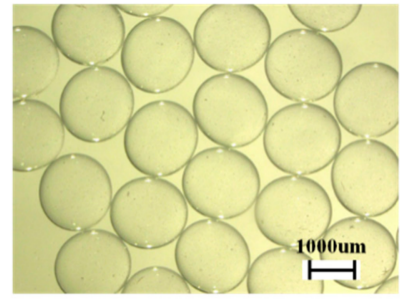

(a)

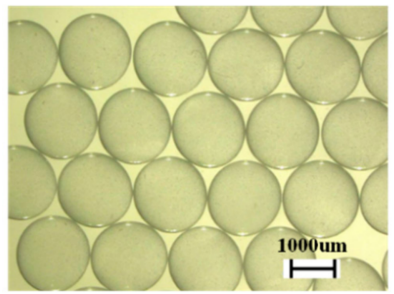

(b)

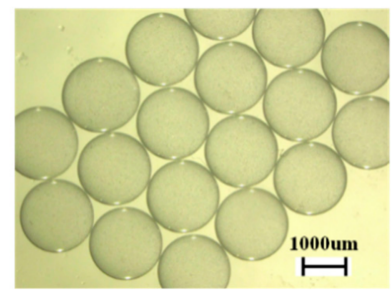

(c)

Figure 10. Hollow microspheres cured by different curing modes. (a) Mode 1; (b) Mode 2; (c) Mode 3.

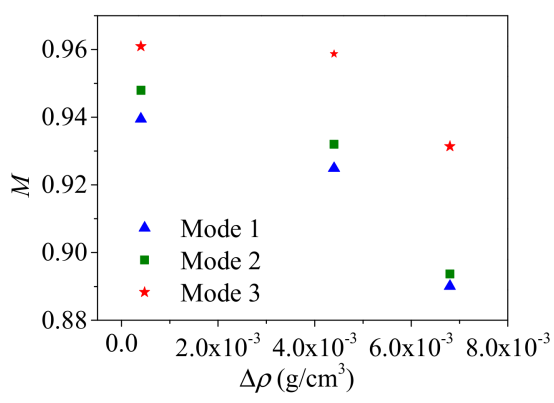

Figure 11. Merit factor of the microsphere for different modes.

\subsection{Optimization of Rotation Rate for the Random Rotating Device}

The effect of the rotation rate on the survival rate, sphericity and uniformity thickness of the hollow microspheres is further studied for the random rotating curing device. First, the survival rates of the microspheres $S$ under rotation rates of 50,100, 150 and $200 \mathrm{r} / \mathrm{min}$ are analyzed and shown in Figure 12. The survival rate of the microspheres first increased and then decreased with the increase of the rotation rate. When the rotation rate is $50 \mathrm{r} / \mathrm{min}$, the fluid movement is so slow that the shear force does not contribute greatly to the movement of the compound droplets, which results in the compound droplets being deposited at the bottom of the container due to gravity and adhering to each other. The increase in the rotation rate enhances the shear force, which increases the survival rate by improving the dispersion uniformity. When the rotation rate continues to increase to a finite value, the larger deformation of the compound droplet leads to the large pressure difference between the inside and outside of the droplet. The interfacial tension is not sufficient for maintaining the stability of the compound droplets, resulting in the break-up of the compound droplets.

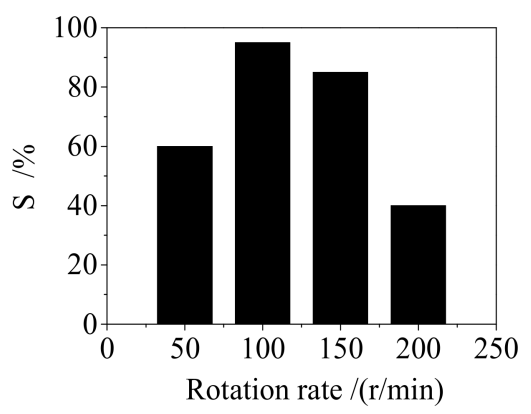

Figure 12. The effect of the rotation rate on the survival rate of the microspheres.

In Figure 13, the cumulative percentages of a sphericity greater than $96.5 \%$ and a wall thickness uniformity under $3 \mu \mathrm{m}$ are given. The results show that the sphericity and thickness uniformity of the microspheres first increased and then decreased as the rotation rate increased. When the rotation rate is low, the effect of the external flow field on the deformation recovery of the hollow microspheres is weak. Therefore, when the rotation rate increases, the appropriate shear force is beneficial to the 
deformation of the compound droplets, which increases the sphericity and thickness uniformity of the hollow microspheres. However, as the rotation rate increase to a large value, the deformation caused by the shear force is too large to recover by the surface tension, resulting in the poor quality of the hollow microspheres. For the rotation rate on the microsphere quality in the random rotating device, the comprehensive quality factor is given as:

$$
Q_{R}=S \times P_{\mathrm{s}} \times P_{\mathrm{m}}
$$

where $S$ is the survival rate, $P_{\mathrm{s}}$ represents the cumulative percentages of a sphericity greater than $96.5 \%$, and $P_{\mathrm{m}}$ represents the cumulative percentages of a wall thickness uniformity under $3 \mu \mathrm{m}$.

Figure 14 shows the effect of the rotation rate on the comprehensive quality factor of the microspheres. The comprehensive quality factor of the microspheres increases with the rotation rate when the rotation rate is lower than $160 \mathrm{r} / \mathrm{min}$. For the random rotating system, the optimal rotation rate is around $160 \mathrm{r} / \mathrm{min}$. However, the comprehensive quality factor of the microspheres reaches nearly zero when the rotation rate is $200 \mathrm{r} / \mathrm{min}$.

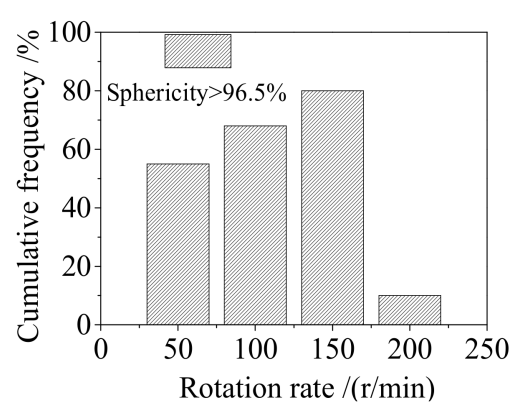

(a)

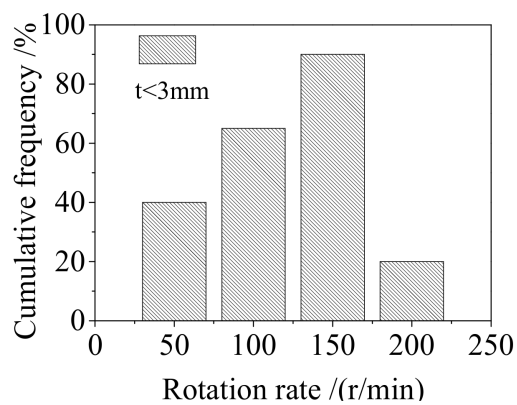

(b)

Figure 13. Effect of the rotation rate on the sphericity and thickness uniformity of the microspheres. (a) sphericity; (b) uniformity of wall thickness.

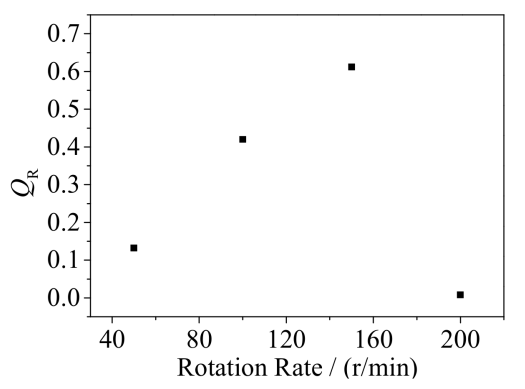

Figure 14. Effect of the rotation rate on the comprehensive quality factor of the microspheres.

\section{Conclusion}

To improve the effect of the external flow fields on the curing quality of the dispersed polystyrene (PS) compound droplets, a new random rotating curing system is designed and experimentally studied via the comparison of the curing process of the PS compound droplets in this new system and that in the traditional rotating curing system. In addition, the effects of the rotation rate, the liquid level and the density difference on the quality of the hollow microspheres are also studied to reveal the mechanism of the rotating curing system. The results indicate that:

- In the random rotating curing system, the disturbance of the fluid increases the deformation recovery ability of the compound droplets. Furthermore, the vortex with different directions in the external flow fields makes the compound droplets spin in many directions, which improves the spheroidization and concentricity of the compound droplets. 
- For the traditional rotating curing system, there is an optimal rotation rate for the best quality of the hollow microspheres, and the optimal rotation rate increases as the liquid level increases.

- Compared with the use of the traditional rotating curing system, the sensitivity of the microspheres quality to the density mismatch between the phases, when utilizing the random rotating curing system, is smaller, and the thickness uniformity and sphericity of the polystyrene (PS) microsphere increase by $10.2 \%$ and $3.6 \%$, respectively.

- There is an optimal rotation rate for the random rotating curing device, which can optimize the survival rate and quality of the hollow microspheres.

Author Contributions: Supervision, F.Y. and C.Y.; Software, Y.Z. and L.W.; Investigation, H.Z. and X.H.

Funding: This research was funded by National Natural Science Foundation of China (Nos. 51706194 and 51876184 ), the China Postdoctoral Science Foundation (2017M621835), and Jiangsu Planned Projects for Postdoctoral Research Funds (1701188B).

Conflicts of Interest: The authors declare no conflict of interest.

\section{References}

1. Zhang, C.; Yu, F.; Li, X.; Chen, Y. Gravity-capillary evaporation regimes in microgrooves. AIChE J. 2019, 65, 1119-1125. [CrossRef]

2. Song, H.; Chen, D.L.; Ismagilov, R.F. Reactions in droplets in microfluidic channels. Angewv. Chem. Int. Ed. 2006, 45, 7336-7356. [CrossRef] [PubMed]

3. Hadwen, B.; Broder, G.R.; Morganti, D.; Jacobs, A.; Brown, C.; Hector, J.R.; Kubota, Y.; Morgan, H. Programmable large area digital microfluidic array with integrated droplet sensing for bioassays. Lab Chip 2012, 12, 3305-3313. [CrossRef] [PubMed]

4. Wang, J.; Sun, L.; Zou, M.; Gao, W.; Liu, C.; Shang, L.; Gu, Z.; Zhao, Y. Bioinspired shape-memory graphene film with tunable wettability. Sci. Adv. 2017, 3, e1700004. [CrossRef] [PubMed]

5. Zhang, C.; Gao, W.; Zhao, Y.; Chen, Y. Microfluidic generation of self-contained multicomponent microcapsules for self-healing materials. Appl. Phys. Lett. 2018, 113, 203702. [CrossRef]

6. Lindl, J. Development of the indirect-drive approach to inertial confinement fusion and the target physics basis for ignition and gain. Phys. Plasmas 1995, 2, 3933-4024. [CrossRef]

7. Du, K.; Liu, M.; Wang, T.; He, X.; Wang, Z.; Zhang, J. Recent progress in ICF target fabrication at RCLF. Matter. Radiat. Extrem. 2018, 3, 135-144. [CrossRef]

8. Lan, K.; Liu, J.; Li, Z.; Xie, X.; Huo, W.; Chen, Y.; Ren, G.; Zheng, C.; Yang, D.; Li, S.; et al. Progress in octahedral spherical hohlraum study. Matter. Radiat. Extrem. 2016, 1, 8-27. [CrossRef]

9. Chen, Y.; Gao, W.; Zhang, C.; Zhao, Y. Three-dimensional splitting microfluidics. Lab Chip 2016, 16, 1332-1339. [CrossRef]

10. Al-Abidi, A.A.; Mat, S.; Sopian, K.; Sulaiman, M.Y.; Mohammad, A.T. Numerical study of PCM solidification in a triplex tube heat exchanger with internal and external fins. Int. J. Heat Mass Transf. 2013, 61, 684-695. [CrossRef]

11. Liu, X.; Zhang, C.; Yu, W.; Deng, Z.; Chen, Y. Bubble breakup in a microfluidic T-junction. Sci. Bull. 2016, 61, 811-824. [CrossRef]

12. Chen, Y.; Liu, X.; Shi, M. Hydrodynamics of double emulsion droplet in shear flow. Appl. Phys. Lett. 2013, 102, 051609. [CrossRef]

13. Kubo, U.; Tsubakihara, H. Development of a coating technique for inertial confinement fusion plastic targets. J. Vac. Sci. Technol. A Vac. Surf. Film. 1986, 4, 1134-1137. [CrossRef]

14. Chen, Y.; Deng, Z. Hydrodynamics of a droplet passing through a microfluidic t-junction. J. Fluid Mech. 2017, 819, 401-434. [CrossRef]

15. Peng, H.; Shi, Z.; Wang, W.; Chen, S.; Zhang, Z.; Xu, Z.; Dong, S.; Chen, Y.P.; Li, B.; Ge, L. An easily assembled double T-shape for the preparation of submillimeter-sized hollow polyacrylonitrile (PAN) microcapsules for inertial confinement fusion (ICF) project. Colloids Surf. A Physicochem. Eng. Asp. 2015, 482, 58-67. [CrossRef] 
16. Chen, S.; Su, L.; Liu, Y.; Li, B.; Qi, X.; Zhang, Z.; Liu, M. Density match during fabrication process of poly ( $\alpha$-methylstyrene) mandrels by microencapsulation. High Power Laser Part. Beams 2012, 24, 1561-1565. [CrossRef]

17. Chen, S.; Liu, Y.; Su, L.; Qi, X.; Shi, R.; Liu, M.; Zhang, Z.; Li, B. Influence of double emulsions solidification rate on sphericity of poly( $\alpha$-methyl styrene) capsules in microencapsulation process. CIESC J. 2013, 64, 2446-2452. (In Chinese)

18. Takagi, M.; Cook, R.; McQuillan, B.; Elsner, F.; Stephens, R.; Nikroo, A.; Gibson, J.; Paguio, S. Development of high quality poly( $\alpha$-methylstyrene) mandrels for NIF. Fusion Sci. Technol. 2002, 41, 278-285. [CrossRef]

19. McQuillan, B.W.; Greenwood, A. Microencapsulation process factors which influence the sphericity of $1 \mathrm{~mm}$ o.d. poly( $\alpha$-methylstyrene) shells for ICF. Fusion Technol. 1999, 35, 194-197. [CrossRef]

20. Mcquillan, B.W.; Nikroo, A.; Steinman, D.A.; Elsner, F.H.; Czechowicz, D.G.; Hoppe, M.L.; Sixtus, M.; Miller, W.J. The PAMS/GDP process for production of ICF target mandrels. Fusion Sci. Technol. 1997, 31, 381-384. [CrossRef]

21. Liu, M.; Chen, S.; Qi, X.; Li, B.; Shi, R.; Liu, Y.; Chen, Y.; Zhang, Z. Improvement of wall thickness uniformity of thick-walled polystyrene shells by density matching. Chem. Eng. J. 2014, 241, 466-476. [CrossRef]

22. Peng, H.; Liu, M.; Li, J.; Chen, S.; Zhang, Z.; Li, B.; Ge, L. Fabrication of thick-walled polyacrylonitrile (PAN) with high uniformity by an easily assembled double-T droplet generator. RSC Adv. 2015, 5, 98892-98899. [CrossRef]

23. Chen, Q.; Chen, S.; Liu, M.; Pan, D.; Li, B.; Zhang, Z.; Qi, X. Influence of fluorobenzene mass transfer on the qualities of poly- $\alpha$-methylstyrene shells. RSC Adv. 2018, 8, 3687-3693. [CrossRef]

24. Pan, D.; Huang, W.; Chen, Q.; Chen, S.; Zhang, Z.; Liu, M.; Li, B. Investigation of craze and cracks of polystyrene shells during drying process. Fusion Sci. Technol. 2018, 73, 59-67. [CrossRef]

25. Liu, M.; Liu, Y.; Li, J.; Chen, S.; Li, J.; Su, L.; Qi, X.; Li, B.; Zhang, Z. Improvement of sphericity of thick-walled polystyrene shell. Colloids Surf. A Physicochem. Eng. Asp. 2015, 484, 463-470. [CrossRef]

(C) 2019 by the authors. Licensee MDPI, Basel, Switzerland. This article is an open access article distributed under the terms and conditions of the Creative Commons Attribution (CC BY) license (http://creativecommons.org/licenses/by/4.0/). 